\title{
THE LOGIC OF BELIEF PERSISTENCE
}

\author{
Pierpaolo Battigalli \\ Princeton University \\ Giacomo Bonanno \\ University of California
}

\section{INTRODUCTION}

The principle of belief persistence, or conservativity principle, states that 'When changing beliefs in response to new evidence, you should continue to believe as many of the old beliefs as possible' (Harman, 1986, p. 46). In particular, this means that if an individual gets new information, she has to accommodate it in her new belief set (the set of propositions she believes), and, if the new information is not inconsistent with the old belief set, then (1) the individual has to maintain all the beliefs she previously had and (2) the change should be minimal in the sense that every proposition in the new belief set must be deducible from the union of the old belief set and the new information (see, e.g., Gärdenfors, 1988; Stalnaker, 1984). We focus on this minimal notion of belief persistence and characterize it both semantically and syntactically.

A 'possible world' semantic formalization of the principle easily comes to mind. The set of all the propositions that the individual believes corresponds to the set of states of the world that she considers possible and is a subset of the set of states that are not ruled out by the individual's information (or knowledge). It is required that, if the individual considers a state possible and her new information does not exclude this state, then she continue to consider it possible. Furthermore, if the individual regards a particular state as impossible, then she should continue to regard it as impossible unless her new information excludes all the states that she previously regarded as possible. This is closely related to the

We thank two anonymous referees, Philippe Mongin and especially Tim Van Zandt for very helpful comments and suggestions. The usual disclaimer applies. 
well-known conditionalization rule to update probability measures. If an individual has probabilistic beliefs, the set of states that she considers possible is simply the support of her subjective probability measure. Let $P_{0}$ be the probability measure representing the agent's beliefs before she receives information $E$ and $P_{n}$ her subjective probability measure after she learns $\mathrm{E}$. The 'qualitative part' of the conditionalization rule states the following:

(C) if $\operatorname{supp}\left(P_{o}\right) \cap E \neq \emptyset$, then $\operatorname{supp}\left(P_{n}\right)=\operatorname{supp}\left(P_{o}\right) \cap E$, where supp $(P)$ denotes the support of the probability measure $P .{ }^{1}$

Despite the apparent simplicity of the semantic formalization, a syntactic characterization of this minimal notion of belief persistence is not obvious. One would like to state something like the following: if the new evidence does not contradict the conjunction of all the propositions believed by the individual, then she incorporates this new evidence in her belief set while maintaining all her previous beliefs. But the belief set is typically infinite and in standard propositional (modal) logic the infinite conjunction of formulae is not a well-formed formula.

We get around this problem by considering an axiom system with knowledge and belief operators for each date satisfying standard properties, including the entailment axiom 'knowledge implies belief'. Given this, we are able to characterize persistence of beliefs with the following axiom which involves only the belief operators:

(PB) The individual believes $\phi$ at date $t$ if and only if she believes at date $t$ that she will believe $\phi$ at date $t+1$.

In Section 2 we begin by studying belief change from a semantic point of view. We consider a class of structures or frames that represent the paradigm in the economics of information literature. There is a countable set of dates. At each date knowledge is represented by a partition of the set of states. Beliefs are based on knowledge, in the sense that the set of states that the individual considers possible at a state $\omega$ is a subset of the cell of the information partition that contains $\omega$. Furthermore, within each cell of the information partition beliefs are the same at every state (that is, the individual knows her own beliefs). We show that the belief persistence condition can be split into two independent properties. The first property is equivalent to the requirement that if the individual at date $t+1$ believes $\phi$, then there be a $\psi$ which she knows at $t+1$ and which at date $t$ she believed to imply $\phi$ (Proposition 2). The second property is

1 The 'quantitative part' states that, for any two subsets $A, B \subseteq$ supp $\left(P_{o}\right) \cap E$, the relative likelihood of $A$ versus $B$ does not change, i.e., $P_{n}(A) P_{0}(B)=P_{0}(A) P_{n}(B)$. There is an ongoing debate in the philosophical literature as to whether or not conditionalization is a requirement of rationality: see, for example, Brown (1976), Jeffrey (1983), Howson and Urbach (1989), Maher (1993) and Teller (1973). 
equivalent to the condition that, if what is known at date $t+1$ does not contradict what is believed at date $t$, then everything which is believed at date $t$ should still be believed at date $t+1$ (Proposition 3). From these two propositions the main result of Section 2 follows, namely that the qualitative part of the conditionalization rule is equivalent to the requirement that, if the new information is consistent with the old beliefs, then the set of propositions that are believed at date $t+1$ be the deductive closure of the union of the set of propositions believed at time $t$ and the set of propositions known at time $t+1$ (Corollary 1 ).

In Section 3 we turn to a syntactic analysis, employing the language of modal logic, which was first used in an epistemic context by the philosopher Hintikka (1962) and whose usefulness has recently been recognized by economists and game theorists. ${ }^{2}$ The formal language that we consider is the one that comes closest to the dynamic models developed in the information economics literature. In particular, time enters the analysis explicitly only through the epistemic operators. The main result of this section (Proposition 5) states that the system obtained by adding axiom (PB) to standard axioms for knowledge and belief and their interaction is sound and complete with respect to the class of models based on frames that satisfy property (C).

Section 4 discusses the notion of perfect recall and the possibility of relaxing the truth axiom for knowledge. Section 5 considers related literature.

\section{THE SEMANTICS OF BELIEF CHANGE}

We shall consider a class of structures that represent the paradigm in the economics of information literature. ${ }^{3}$ Let $\Omega$ be a (nonempty and possibly infinite) set of states. At every date $t \in \mathbb{N}$ (where $\mathbb{N}$ denotes the set of natural numbers), ${ }^{4}$ the individual has a partition of the set $\Omega$, representing her information or knowledge. We represent this partition by means of an information correspondence $\mathcal{K}_{t}: \Omega \rightarrow 2^{\Omega}$ [where $2^{\Omega}$ is the set of events, that is, subsets of $\Omega$ ] that satisfies the following properties: $\forall t \in \mathbb{N}, \forall \omega, \omega^{\prime} \in \Omega$

reflexivity: $\omega \in \mathcal{K}_{t}(\omega)$

transitivity: if $\omega^{\prime} \in \mathcal{K}_{\mathrm{t}}(\omega)$ then $\mathcal{K}_{\mathrm{t}}\left(\omega^{\prime}\right) \subseteq \mathcal{K}_{\mathrm{t}}(\omega)$

2 For recent contributions and a list of references see the special issue of Theory and Decision on 'Logic and the epistemic foundations of game theory' (1994, Vol. 37). See also the recent survey by Dekel and Gul (1996).

${ }^{3}$ See, for example, Geanakoplos (1994, pp. 1456-8). For the importance of the interaction between knowledge and belief in game theory see Dekel and Gul (1996).

${ }^{4}$ We model time as discrete and take the set of dates to be $\mathbb{N}$. The latter assumption is without loss of generality since the case of a finite number of dates can be modeled by assuming that there are no changes in information and beliefs after a certain date. 
euclideanness: if $\omega^{\prime} \in \mathcal{K}_{\mathrm{t}}(\omega)$ then $\mathcal{K}_{\mathrm{t}}(\omega) \subseteq \mathcal{K}_{\mathrm{t}}\left(\omega^{\prime}\right) .{ }^{5}$

For every state $\omega \in \Omega, \mathcal{K}_{t}(\omega)$ is the set of states that, according to her information, the individual cannot rule out at $\omega$.

To represent the individual's beliefs, we define, for every date $t$, a belief correspondence $\mathcal{B}_{\mathrm{t}}: \Omega \rightarrow 2^{\Omega}$ that satisfies the following properties: $\forall t \in \mathbb{N}, \forall \omega, \omega^{\prime} \in \Omega$

seriality: $\mathcal{B}_{\mathrm{t}}(\omega) \neq \varnothing$

transitivity: if $\omega^{\prime} \in \mathcal{B}_{\mathrm{t}}(\omega)$ then $\mathcal{B}_{\mathrm{t}}\left(\omega^{\prime}\right) \subseteq \mathcal{B}_{\mathrm{t}}(\omega)$

euclideanness: if $\omega^{\prime} \in \mathcal{B}_{\mathrm{t}}(\omega)$ then $\mathcal{B}_{\mathrm{t}}(\omega) \subseteq \mathcal{B}_{\mathrm{t}}\left(\omega^{\prime}\right)$.

Thus $\mathcal{B}_{\mathfrak{t}}(\omega)$ is the set of states that the individual considers possible at $\omega$. Furthermore, beliefs are based on information and depend only on it, in the following sense: $\forall t \in \mathbb{N}, \forall \omega, \omega^{\prime} \in \Omega$

(R1) $\quad \mathcal{B}_{\mathrm{t}}(\omega) \subseteq \mathcal{K}_{\mathrm{t}}(\omega)$

(R2) if $\omega^{\prime} \in \mathcal{K}_{\mathrm{t}}(\omega)$ then $\mathcal{B}_{\mathrm{t}}\left(\omega^{\prime}\right)=\mathcal{B}_{\mathrm{t}}(\omega)$.

It will be shown below (Remark 1) that (R1) corresponds to the requirement that the individual always believe what she knows and (R2) to the requirement that the individual know her own beliefs. ${ }^{6}$

Whenever $\Omega$ is a non-empty set, $\mathcal{K}_{t}: \Omega \rightarrow 2^{\Omega}$ is reflexive, transitive and euclidean, $\mathcal{B}_{\mathrm{t}}: \Omega \rightarrow 2^{\Omega}$ is serial, transitive and euclidean and together they satisfy (R1) and (R2), we call the structure $\left\langle\Omega,\left\{\mathcal{K}_{\mathrm{t}}\right\}_{\mathrm{t} \in \mathrm{Ni}}\left\{\mathcal{B}_{\mathrm{t}}\right\}_{\mathrm{t} \in \mathbb{N}}\right\rangle$, a $K B$ frame ('KB' stands for 'Knowledge and Belief'). We denote by $\mathcal{F}_{\mathrm{KB}}$ the set of KB-frames.

Belief and knowledge pertain to propositions. Events, that is, subsets of $\Omega$ should be thought of as representing propositions. In order to establish the interpretation of events as propositions we need to introduce the notion of a model based on a frame.

We consider a language with two operators for every $t \in \mathbb{N}$ : $B_{t}$ and $K_{t}$. The intended interpretation of $\mathrm{B}_{\mathrm{t}} \phi$ is 'at time $t$ the individual believes $\phi$ ' and the interpretation of $K_{t} \phi$ is 'at time $t$ the individual knows $\phi$ '. The alphabet of the language consists of: (1) a finite or countable set $\Pi$ of

5 Transitivity is made redundant by reflexivity and euclideanness (see Chellas, 1984, p. 85). However, throughout the paper we shall allow for some redundancies when they add clarity to the exposition or make things look more familiar in the light of the existing literature.

6 In the economics of information literature beliefs are usually represented by a collection of probability measures, one for each cell of the information partition. In this case one can interpret $\mathcal{B}_{\ell}(\omega)$ as the support of the probability measure over the cell of the partition that contains state $\omega$. It is easy to verify that, with this interpretation, the belief correspondence $\mathcal{B}_{t}$ indeed satisfies seriality, transitivity and euclideanness, as well as (R1) and (R2). See also Halpern (1991). 
sentence letters (representing atomic propositions), ${ }^{7}(2)$ the connectives $\neg$ (for 'not'), $\vee$ (for 'or'), and, for every $t \in \mathbb{N}, B_{t}$ and $K_{t},(3)$ the bracket symbols ( and ). The set $\Phi$ of formulae is obtained from the sentence letters by closing with respect to negation, disjunction and the operators $B_{t}$ and $\mathrm{K}_{\mathrm{t}}{ }^{8}$ As is customary, we shall often omit the outermost brackets (e.g., we shall write $\phi \vee \psi$ instead of $(\phi \vee \psi)$ ) and use the following (metalinguistic) abbreviations: $\phi \wedge \psi$ for $\neg(\neg \phi \vee \neg \psi)$ (the symbol $\wedge$ stands for 'and'), $\phi \rightarrow \psi$ for $(\neg \phi) \vee \psi$ (the symbol $\rightarrow$ stands for 'if . . . then . . .') and $\phi \leftrightarrow \psi$ for $(\phi \rightarrow \psi) \wedge(\psi \rightarrow \phi)$ (the symbol $\leftrightarrow$ stands for 'if and only if').

Given a frame $\mathrm{F} \in \mathcal{F}_{\mathrm{KB}}$ one obtains a model based on it by adding a function $f: \Pi \rightarrow 2^{\Omega}$ that associates with every sentence letter $\pi$ the set of states at which $\pi$ is true. For every formula $\phi \in \Phi$, the truth set of $\phi$ in $\mathscr{M}$, denoted by $\|\phi\|^{\mathscr{H}}$, is defined recursively as follows:

(1) If $\phi=(\pi)$ where $\pi$ is a sentence letter, then $\|\phi\|^{\mathscr{A}}=f(\pi)$,

(2) $\|\neg \phi\|^{\mathscr{A}}=-\|\phi\|^{\mathscr{A}}$ (where '-' denotes complement)

(3) $\|\phi \vee \psi\|^{\mathscr{H}}=\|\phi\|^{\mathscr{M}} \cup\|\psi\|^{\mathscr{M}}$,

(4) For all $t \in \mathbb{N}$

$$
\begin{aligned}
& \left\|K_{t} \phi\right\|^{\mathscr{M}}=\left\{\omega \in \Omega: \mathcal{K}_{\mathrm{t}}(\omega) \subseteq\|\phi\|^{\mathscr{H}}\right\}, \text { and } \\
& \left\|B_{\mathrm{t}} \phi\right\|^{\mathscr{A}}=\left\{\omega \in \Omega: \mathcal{B}_{\mathrm{t}}(\omega) \subseteq\|\phi\|^{\mathscr{M}}\right\} .9
\end{aligned}
$$

If $\omega \in\|\phi\|^{\mathscr{M}}$ we say that $\phi$ is true at state $\omega$ in model $\mathscr{M}$. An alternative notation for $\omega \in\|\phi\|^{\mathscr{A}}$ is $\models_{\omega}^{\mathscr{A}} \phi$ and an alternative notation for $\omega \notin\|\phi\|^{\mathscr{H}}$ is $\nvdash_{\omega}^{\mathscr{A}} \phi$. A formula $\phi$ is valid in model $\mathscr{M}$ if and only if $\models_{\omega}^{\mathscr{H}} \phi$ for all $\omega \in \Omega$.

For every $\mathrm{F} \in \mathcal{F}_{\mathrm{KB}}$ we denote by $\mathrm{M}(\mathrm{F})$ the set of models based on $\mathrm{F}$.

REMARK 1. Let $\mathrm{F} \in \mathcal{F}_{\mathrm{KB}}$ and $\mathscr{M} \in \mathbb{M}(\mathrm{F})$. Then, for every $t \in \mathbb{N}$ and $\phi \in \Phi$, by (R1), the formula $K_{t} \phi \rightarrow B_{t} \phi$ is valid in $\mathscr{M}$, and, by (R2), the formula $\mathrm{B}_{\mathrm{t}} \phi \rightarrow \mathrm{K}_{\mathrm{t}} \mathrm{B}_{\mathrm{t}} \phi$ is valid in $\mathscr{M}^{10}$

Within our framework, the qualitative part of the conditionalization rule can be restated as follows:

${ }^{7}$ Each sentence letter $\pi \in \Pi$ should be interpreted as a partial description of the world at a specific point in time (and location). Different sentence letters may refer to different times and location.

${ }^{8}$ Thus $\Phi$ is obtained recursively as follows: (i) for every sentence letter $\pi,(\pi) \in \Phi$, (ii) if $\phi, \psi \in \Phi$ then $(\neg \phi) \in \Phi,(\phi \vee \psi) \in \Phi$ and, for every $t \in \mathbb{N},\left(B_{t} \phi\right) \in \Phi$ and $\left(K_{\imath} \phi\right) \in \Phi$.

${ }^{9}$ Thus at state $\omega$ (and time $t$ ) the individual believes $\phi$ if and only if $\phi$ is true at all the states that she considers possible at $\omega$. Mongin (1994) points out the awkwardness of this (standard) informal explication of Kripke structures and argues in favor of a semantics based on neighborhood structures.

${ }^{10}$ We comment on the axiom schemata $K_{t} \phi \rightarrow B_{t} \phi$ and $B_{t} \phi \rightarrow K_{t} B_{t} \phi$ in the next section. Remark 1 can be verified directly. It is also a consequence of Theorem 4.3 in van der Hoek (1993, p. 183). We are grateful to Joe Halpern for bringing this paper to our attention. 
(C) $\forall t \in \mathbb{N}, \forall \omega \in \Omega$, if $\mathcal{B}_{t}(\omega) \cap \mathcal{K}_{t+1}(\omega) \neq \emptyset$ then $\mathcal{B}_{t+1}(\omega)=\mathcal{B}_{t}(\omega) \cap \mathcal{K}_{t+1}(\omega)$.

The main purpose of this paper is to provide a characterization and axiomatization of (C). Given (R1), (C) can be split into two parts, (P1) and (P2), where

(P1) $\forall \omega \in \Omega, \forall t \in \mathbb{N}, \quad \mathcal{B}_{\mathrm{t}}(\omega) \cap \mathcal{K}_{\mathrm{t}+1}(\omega) \subseteq \mathcal{B}_{\mathrm{t}+1}(\omega)$.

(P2) $\forall \omega \in \Omega, \forall t \in \mathbb{N}$, if $\mathcal{B}_{\mathfrak{t}}(\omega) \cap \mathcal{K}_{t+1}(\omega) \neq \emptyset$ then $\mathcal{B}_{\mathrm{t}+1}(\omega) \subseteq \mathcal{B}_{\mathfrak{t}}(\omega)$.

Propositions 1-3 below provide two different characterizations of (P1) and (P2). All the proofs are given in the appendix.

PROPOSITION 1. Fix an arbitrary frame $F \in \mathcal{F}_{\text {KB }}$. Then:

(1) F satisfies (P1) if and only if

$$
\forall \mathscr{M} \in \mathbb{M}(\mathrm{F}), \forall t \in \mathbb{N}, \forall \omega \in \Omega, \forall \phi \in \Phi, \quad F_{\omega}^{\mathscr{\mu}}\left(\mathrm{B}_{\mathrm{t}} \mathrm{B}_{\mathrm{t}+1} \phi \rightarrow \mathrm{B}_{\mathrm{t}} \phi\right) ;
$$

(2) F satisfies (P2) if and only if

$$
\forall \mathscr{M} \in \mathbb{N}(\mathrm{F}), \forall t \in \mathbb{N}, \forall \omega \in \Omega, \forall \phi \in \Phi, \quad \models_{\omega}^{\mathscr{H}}\left(\mathrm{B}_{\mathrm{t}} \phi \rightarrow \mathrm{B}_{\mathrm{t}} \mathrm{B}_{\mathrm{t}+1} \phi\right) .
$$

Thus, condition (C) holds in a frame $F \in \mathcal{F}_{\mathrm{KB}}$ if and only if the formula $B_{t} \phi \leftrightarrow B_{t} B_{t+1} \phi$ (which says that at date $t$ the individual believes $\phi$ if and only if she believes that at date $t+1$ she will believe $\phi)$ is valid in every model based on F. Note that this formula involves only the belief operators, while $(C)$ is a condition that involves both the belief and the knowledge correspondences. This reduction of $(C)$ to a condition only on beliefs is possible because of the interaction between knowledge and belief imposed by (R1) and (R2) (cf. Remark 1).

In order to obtain a further characterization of $(C)$ we introduce the notions of consistency, belief set and knowledge set.

DEFINITION 1. A set of formulae $S \subseteq \Phi$ is consistent if, for every formula $\phi$,

$$
\phi \in \mathrm{S} \Rightarrow \neg \phi \notin \mathrm{S} .
$$

DEFINITION 2. Given a model $\mathscr{M}$, for every $t \in \mathbb{N}$ and $\omega \in \Omega$ let

$$
\begin{gathered}
\beta^{\mathscr{M}}(t, \omega)=\left\{\phi \in \Phi: \models_{\omega}^{\mathscr{\mu}} \mathrm{B}_{\mathrm{t}} \phi\right\} \text { and } \\
\kappa^{\mathscr{H}}(t, \omega)=\left\{\phi \in \Phi: \models_{\omega}^{\mathscr{\mu}} \mathrm{K}_{\mathrm{t}} \phi\right\} .
\end{gathered}
$$

Thus $\phi \in \beta^{\mathscr{\mu}}(t, \omega)$ if and only if $\mathcal{B}_{t}(\omega) \subseteq\|\phi\|^{\mu}$. Similarly, $\phi \in \kappa^{\mu}(t, \omega)$ if and only if $\mathcal{K}_{t}(\omega) \subseteq\|\phi\|^{\mathscr{\mu}}$. We call $\beta^{\mathscr{\mu}}(t, \omega)$ the individual's belief set at date $t$ and state $\omega$ in model $\mathscr{M}$ and $\kappa^{\mathscr{H}}(t, \omega)$ her knowledge set (at $t$ and $\omega$ ). Note that, for every model $\mathscr{M}$ based on a frame $\mathrm{F} \in \mathcal{F}_{\mathrm{KB}}$, and for all $t \in \mathbb{N}$ 
and $\omega \in \Omega, \beta^{\mathscr{H}}(t, \omega)$ and $\kappa^{\mathscr{H}}(t, \omega)$ are consistent, since $\mathcal{B}_{\mathrm{t}}$ and $\mathcal{K}_{\mathrm{t}}$ are serial (the latter is reflexive, hence serial). ${ }^{11}$

Proposition 2 below says that (P1) is equivalent to the requirement that, moving from date $t$ to date $t+1$, every new belief be deducible from the knowledge acquired at date $t+1$ and the beliefs at date $t$.

PROPOSITION 2. Fix an arbitrary frame $F \in \mathcal{F}_{\mathrm{KB}}$. Then the following are equivalent:

(1) F satisfies (P1),

(2) $\forall \mathscr{M} \in \mathbb{M}(\mathrm{F}), \forall t \in \mathbb{N}, \forall \omega \in \Omega, \forall \phi \in \Phi$,

if $\phi \in \beta^{\mathscr{A}}(t+1, \omega)$ then there exists a $\psi \in \kappa^{\mathscr{M}}(t+1, \omega)$ such that $(\psi \rightarrow \phi) \in \beta^{\mathscr{M}}(t, \omega)$,

(that is, if the individual at date $t+1$ believes $\phi$, then there must be something which she knows at $t+1$ and which at date $t$ she believed to imply $\phi$ ).

Thus, by Proposition 2, condition (P1) is equivalent to ruling out 'arbitrary' expansions of the belief set. Proposition 3 below, on the other hand, shows that (P2) is equivalent to ruling out 'arbitrary' contractions of the belief set: imposing (P2) amounts to requiring that, if what is known at date $t+1$ is consistent with what was believed at date $t$, then at date $t+1$ the individual continue to believe everything that she believed at date $t$.

PROPOSITION 3. Fix an arbitrary frame $F \in \mathcal{F}_{\mathrm{KB}}$. Then the following are equivalent:

(1) F satisfies (P2),

(2) $\forall \mathscr{M} \in \mathbb{M}(\mathrm{F}), \forall t \in \mathbb{N}, \forall \omega \in \Omega$,

if $\beta^{\mathscr{H}}(t, \omega) \cup \kappa^{\mathscr{H}}(t+1, \omega)$ is consistent then $\beta^{\mathscr{H}}(t, \omega) \subseteq \beta^{\mathscr{H}}(t+1, \omega)$,

(that is, if what is known at date $t+1$ does not contradict what is believed at date $t$, then everything which is believed at date $t$ is still believed at date $t+1)$.

DEFINITION 3. Given a set of formulae $S \subseteq \Phi$, its $P L$-deductive closure $[S]^{\mathrm{PL}}$ (where 'PL' stands for 'Propositional Logic') is defined as follows: $\psi \in[S]^{\mathrm{PL}}$ if and only if there exist $\phi_{1}, \ldots, \phi_{\mathrm{n}} \in \mathrm{S}$ such that $\left(\phi_{1} \wedge \ldots \wedge \phi_{n}\right)$ $\rightarrow \psi$ is a propositional tautology (that is, a theorem of Propositional Logic).

It is well-known (see Chellas, 1984) that for every model $\mathscr{M}$ based on

${ }^{11}$ It can be shown (see, in particular, the remark following Definition 3 ) that $\beta^{\mu \prime}(t, \omega)$ is a belief set in the sense of Gärdenfors (1988, p. 24); it also coincides with an 'acceptance state' as defined by Stalnaker (1984, pp. 81-2). The same holds for $\kappa^{\mu \prime}(t, \omega)$. 
a frame $\mathrm{F} \in \mathcal{F}_{\mathrm{KB}}$, for every $t \in \mathbb{N}$ and $\omega \in \Omega, \beta^{\mathscr{\mu}}(t, \omega)=\left[\beta^{\mathscr{\mu}}(t, \omega)\right]^{\mathrm{PL}}$ and $\boldsymbol{\kappa}^{\mathscr{M}}(t, \omega)=\left[\kappa^{\mathscr{N}}(t, \omega)\right]^{\mathrm{PL}}$.

The following corollary is the main result of this section. ${ }^{12}$ It states that a KB-frame satisfies condition (C) if and only if - for every model based on it - if the union of the old belief set and the new knowledge set is consistent then its PL-deductive closure coincides with the new belief set.

COROLLARY 1. Fix an arbitrary frame $F \in \mathcal{F}_{\text {KB }}$. Then the following are equivalent:

(1) F satisfies (C),

(2) $\forall \mathscr{M} \in \mathbb{N}(\mathrm{F}), \forall t \in \mathbb{N}, \forall \omega \in \Omega$,

if $\beta^{\mathscr{M}}(t, \omega) \cup \kappa^{\mathscr{H}}(t+1, \omega)$ is consistent then $\beta^{\mathscr{\mu}}(t+1, \omega)=\left[\beta^{\mathscr{\mu}}(t, \omega) \cup\right.$ $\left.\kappa^{\mathscr{H}}(t+1, \omega)\right]^{\mathrm{PL}}$.

We now turn to the syntactic analysis, to provide an axiomatization of (C) within the class of KB-frames.

\section{THE SYNTAX OF BELIEF CHANGE}

Let $\mathrm{K}_{\mathrm{KB}}^{\text {time }}$ be the system or calculus specified by the following axiom schemata and rules of inference:

(1) A suitable axiomatization of propositional calculus,

(2) the following schemata ${ }^{13}$ (the names are as in Chellas, 1984), for every $t \in \mathbb{N}$,

$\left(\mathrm{K}_{\mathrm{K}}\right) \mathrm{K}_{\mathrm{t}}(\phi \rightarrow \psi) \rightarrow\left(\mathrm{K}_{\mathrm{t}} \phi \rightarrow \mathrm{K}_{\mathrm{t}} \psi\right)$,

$\left(K_{B}\right) B_{t}(\phi \rightarrow \psi) \rightarrow\left(B_{t} \phi \rightarrow B_{t} \psi\right)$,

$\left(\mathrm{T}_{\mathrm{K}}\right) \mathrm{K}_{\mathrm{t}} \phi \rightarrow \phi$

(truth or veridicality of knowledge),

$(4 \mathrm{~K}) \mathrm{K}_{\mathrm{t}} \phi \rightarrow \mathrm{K}_{\mathrm{t}} \mathrm{K}_{\mathrm{t}} \phi$ (positive introspection of knowledge)

$\left(5_{\mathrm{K}}\right) \neg \mathrm{K}_{\mathrm{t}} \phi \rightarrow \mathrm{K}_{\mathrm{t}} \neg \mathrm{K}_{\mathrm{t}} \phi$ (negative introspection of knowledge)

$\left(D_{\mathrm{B}}\right) \mathrm{B}_{\mathrm{t}} \phi \rightarrow \neg \mathrm{B}_{\mathrm{t}} \neg \phi$ (consistency of beliefs)

$\left(4_{B}\right) \quad B_{t} \phi \rightarrow B_{t} B_{t} \phi$ (positive introspection of beliefs)

12 We are grateful to Tim Van Zandt for prompting us to prove this result.

${ }^{13}$ The axiomatization given below is not minimal. In particular, the positive and negative introspection axioms for the belief operator are redundant, since they can be derived from the remaining axioms (semantically, if $\mathcal{K}_{t}$ is reflexive and euclidean, $\mathcal{B}_{t}$ is serial and (R1) and (R2) are satisfied, then $\mathcal{B}_{t}$ is transitive and euclidean: cf. Kraus and Lehmann, 1988). Furthermore, it is well known (cf. Chellas, 1984) that the positive introspection axiom for knowledge can be derived from the other two axioms for knowledge. We chose this nonminimal formulation in order to make the discussion of Section 4 easier to follow. 
$\left(5_{\mathrm{B}}\right) \neg \mathrm{B}_{\mathrm{t}} \phi \rightarrow \mathrm{B}_{\mathrm{t}} \neg \mathrm{B}_{\mathrm{t}} \phi$

(A1) $\mathrm{K}_{\mathrm{t}} \phi \rightarrow \mathrm{B}_{\mathrm{t}} \phi$

(A2) $B_{t} \phi \rightarrow K_{t} B_{t} \phi$ (negative introspection of beliefs)

(entailment axiom)

(knowledge of own beliefs)

(3) the rule of inference Modus Ponens:

$$
\operatorname{MP} \frac{\phi, \phi \rightarrow \psi}{\psi}
$$

(4) the rule of inference Necessitation: for every $t \in \mathbb{N}$,

$$
\frac{\phi}{\mathrm{K}_{\mathrm{t}} \phi} \text { and } \frac{\phi}{\mathrm{B}_{\mathrm{t}} \phi} \text {. }
$$

Unlike the other axioms, perhaps (A1) and (A2) are less well-known. (A1) says that the individual believes everything that she knows. It is referred to in the philosophical literature as 'the entailment thesis'. (A2) says that if the individual believes something then she knows that she believes it. Both axioms were first discussed by Hintikka (1962), who defended (A1) but rejected (A2). Lenzen (1978) provides a detailed discussion of these two axioms and refutes attempts to reject them. Concerning the entailment thesis, Lenzen concludes (p. 27) that it is sounder than its critics claim. It may be regarded as a cornerstone of epistemic logic'. Concerning (A2), Lenzen finds Hintikka's rejection surprising; he endorses (A2) after a detailed discussion of the arguments that have been put forward against it. Both axioms are implicit in the economics of information literature, where beliefs are represented by subjective probability measures conditional on the information received. Indeed (A1) corresponds to the requirement that the subjective probability measure assign probability 1 to the information received and (A2) corresponds to the assumption that, within each element of the information partition, the subjective probability measure be independent of the state (cf. Remark 1).

Let $\boldsymbol{\Sigma}$ be an extension of $\mathbf{K}_{\mathbf{K B}}^{\text {time }}$, that is, $\boldsymbol{\Sigma}$ is either $\mathbf{K}_{\mathbf{K B}}^{\text {time }}$ itself or a system obtained by adding one or more axiom schemata to $K_{\mathbf{K B}}^{\text {time }}$. Let $\mathrm{M}$ be a class of models. Then we say that $\Sigma$ is sound with respect to $\mathbb{W}$ if every theorem of $\boldsymbol{\Sigma}$ (that is, every axiom and every formula that can be obtained, in a finite number of steps, from the axioms using the rules of inference ${ }^{14}$ ) is valid in every model in $\mathbb{2}$ and we say that $\boldsymbol{\Sigma}$ is complete with respect to $\mathbb{Q}$ if every formula that is valid in every model in $\mathbb{Q}$ is is a theorem of $\boldsymbol{\Sigma}$.

${ }^{14}$ Formally: $\phi$ is a theorem of $\Sigma$ if and only if there exist $m \geq 1$ formulae $\phi_{1}, \ldots, \phi_{m}$ such that $\phi_{m}=\phi$ and, for every $k \leq m, \phi_{k}$ is either an axiom or is obtained from some $\phi_{i}$ and $\phi_{j}$ (with $i, j<k$ ) by applying one of the rules of inference. 
REMARK 2. Let $\mathbb{Q}\left(\mathcal{F}_{\mathrm{KB}}\right)$ be the class of models based on the frames in $\mathcal{F}_{\mathrm{KB}}$. Then $\mathbf{K}_{\mathrm{KB}}^{\text {time }}$ is sound and complete with respect to $\mathbb{Q}\left(\mathcal{F}_{\mathrm{KB}}\right){ }^{15}$

We can now turn to the main purpose of this section, which is the axiomatization of $(C)$, the qualitative version of the conditionalization rule. Consider the following axiom schemata, which were first introduced in Proposition 1: for all $t \in \mathbb{N}$,

(A3) $B_{t} B_{t+1} \phi \rightarrow B_{t} \phi$

(A4) $\mathrm{B}_{\mathrm{t}} \phi \rightarrow \mathrm{B}_{\mathrm{t}} \mathrm{B}_{\mathrm{t}+1} \phi$

(A3) says that if at date $t$ the individual believes that she will believe $\phi$ at date $t+1$, then she must believe $\phi$ at date $t$. (A4) says the converse: if the individual believes $\phi$ at date $t$, then she must also believe, at date $t$, that she will believe $\phi$ at date $t+1$.

First we show that (A3) and (A4) are equivalent to more general axioms which look more than one date into the future. Consider the following axiom schemata:

$\forall t \in \mathbb{N}, \forall \mathbf{k} \in \mathbb{N}, \mathbf{k} \geq 1$

$\left(\mathrm{A}^{\prime}\right) \mathrm{B}_{\mathrm{t}} \mathrm{B}_{\mathrm{t}+\mathrm{k}} \phi \rightarrow \mathrm{B}_{\mathrm{t}} \phi$

$\left(\mathrm{A} 4^{\prime}\right) \mathrm{B}_{\mathrm{t}} \phi \rightarrow \mathrm{B}_{\mathrm{t}} \mathrm{B}_{\mathrm{t}+\mathrm{k}} \phi$.

$\left(A 3^{\prime}\right)$ says that if the individual believes at date $t$ that she will believe $\phi$ at any future date, then she believes $\phi$ at date $t$, while (A4') says the converse. Clearly, (A3) and (A4) are instances (with $k=1)$ of $\left(A 3^{\prime}\right)$ and $\left(\mathrm{A} 4^{\prime}\right)$, respectively. Let $\mathrm{K}_{\mathrm{KB}}^{\text {time }}+(\mathrm{A} 3)+(\mathrm{A} 4)$ be the system obtained by adding (A3) and (A4) to $\mathrm{K}_{\mathrm{KB}}^{\text {time }}$. By Proposition 4 below, $\mathrm{K}_{\mathrm{KB}}^{\mathrm{time}}+(\mathrm{A3})+(\mathrm{A4})$ is equivalent to the system obtained by adding to $\mathbf{K}_{\mathbf{K B}}^{\text {time }}$ all the instances of $\left(A 3^{\prime}\right)$ and $\left(A 4^{\prime}\right)$ (for the definition of a theorem see Footnote 14).

PROPOSITION 4. Every instance of $\left(A 3^{\prime}\right)$ and $\left(A 4^{\prime}\right)$ is a theorem of $K_{K B}^{\text {time }}$ $+(\mathrm{A} 3)+(\mathrm{A} 4)$.

The following proposition is the main result of this section. It states that the conjunction of (A3) and (A4) provides an axiomatization of (C) within the class of KB-frames. Let $\mathcal{F}_{\mathrm{KB}}^{C}$ be the subset of $\mathcal{F}_{\mathrm{KB}}$ consisting of those frames that satisfy $(C)$ and let $\mathrm{M}\left(\mathcal{F}_{\mathrm{KB}}^{\mathrm{C}}\right)$ be the class of models based on frames in $\mathcal{F}_{\mathrm{KB}}^{\mathrm{C}}$.

PROPOSITION 5. $\mathbf{K}_{\mathbf{K} B}^{\text {time }}+(\mathrm{A} 3)+(\mathrm{A} 4)$ is sound and complete with respect to $\mathrm{M}\left(\mathcal{F}_{\mathrm{KB}}^{\mathrm{C}}\right)$.

${ }^{15}$ This is a straightforward extension (to our temporal framework) of a result by Kraus and Lehmann (1988). See Section 5 for further discussion. 


\section{DISCUSSION}

In the information economics literature it is standard to assume that the individual, as time progresses, never forgets what she knew before and possibly acquires more information. Such an assumption, called 'perfect recall', ${ }^{16}$ is captured semantically by the requirement that the information partition at time $t+1$ be a refinement of the information partition at time $t$ :

(R5) $\forall t \in \mathbb{N}, \forall \omega \in \Omega, \quad \mathcal{K}_{t+1}(\omega) \subseteq \mathcal{K}_{t}(\omega)$

REMARK 3. Let $F \in \mathcal{F}_{\mathrm{KB}}$ be a frame that satisfies (R5). Then the following schema is valid in every model based on F:

(A5) $\mathrm{K}_{\mathrm{t}} \phi \rightarrow \mathrm{K}_{\mathrm{t}+1} \phi$.

Furthermore, if (A5) is added to $K_{K B}^{\text {time }}$ (respectively $K_{K B}^{\text {time }}+(A 3)+(A 4)$ ), then the resulting system is sound and complete with respect to the class of models based on KB-frames that satisfy (R5) (respectively (C) and (R5)). ${ }^{17}$

(A5) says that if the individual knows $\phi$ at date $t$ then she still knows $\phi$ at date $t+1$. There are other axioms, besides (A5), that could be proposed as an expression of the notion of perfect recall. For example, one could find (A6) or (A7) below more appealing:

$$
\begin{aligned}
& \text { (A6) } \neg \mathrm{K}_{\mathrm{t}} \phi \rightarrow \mathrm{K}_{\mathrm{t}+1} \neg \mathrm{K}_{\mathrm{t}} \phi \\
& \text { (A7) } \mathrm{K}_{\mathrm{t}} \phi \rightarrow \mathrm{K}_{\mathrm{t}+1} \mathrm{~K}_{\mathrm{t}} \phi
\end{aligned}
$$

(A6) says that if the individual doesn't know something at date $t$ then at date $t+1$ she knows that she did not know, while (A7) says that if the individual knows something at date $t$ then at date $t+1$ she will know that she knew. Perhaps not surprisingly, the following proposition shows that, granting our basic system, these three axioms are equivalent.

PROPOSITION 6. The following three systems are equivalent:

(1) $K_{K B}^{\text {time }}+(A 5)$, (2) $K_{K B}^{\text {time }}+(A 6)$, and (3) $K_{K B}^{\text {time }}+(A 7)$.

Note that the perfect recall axiom (A5) is not a theorem of $\mathbf{K}_{\mathbf{K B}}^{\text {time }}$ $+(A 3)+(A 4)$ (and neither is belief in perfect recall: $\left.B_{t}\left(K_{t} \phi \rightarrow K_{t+1} \phi\right)\right) \cdot{ }^{18}$ For

${ }^{16}$ In the game theoretic literature this assumption is sometimes called 'perfect recall of information sets', whereas 'perfect recall' refers to a stronger assumption (see, for example, Piccione and Rubinstein, 1995).

17 This result can be obtained as an application of Theorem 4.3 in van der Hoek (1993, p. 183).

${ }^{18}$ Consider the following KB-frame: $\Omega=\{\alpha, \beta, \gamma\}, \mathcal{K}_{0}(\alpha)=\mathcal{K}_{0}(\beta)=\{\alpha, \beta\}, \mathcal{B}_{0}(\alpha)=\mathcal{B}_{0}(\beta)=$ $\{\alpha\}, \mathcal{K}_{0}(\gamma)=\mathcal{B}_{0}(\gamma)=\{\gamma\}$, and, for all $t \geq 1, \mathcal{K}_{\mathrm{t}}(\alpha)=\mathcal{B}_{\mathrm{t}}(\alpha)=\{\alpha\}, \mathcal{K}_{\mathrm{t}}(\beta)=\mathcal{K}_{\mathrm{t}}(\gamma)=\{\beta, \gamma\}$, $\mathcal{B}_{\mathrm{t}}(\beta)=\mathcal{B}_{\mathrm{t}}(\gamma)=\{\gamma\}$. This KB-frame satisfies $(C)$. Let $\mathscr{M}$ be a model based on this frame where $\|\pi\|=\{\gamma\}$. Then both $K_{0} \pi \rightarrow K_{1} \pi$ and $B_{0}\left(K_{0} \pi \rightarrow K_{1} \pi\right)$ are false at $\gamma$. Thus, by 
the sake of parsimony, the results of Sections 2 and 3 were obtained without assuming perfect recall. But in a context in which perfect recall is not assumed, condition $(C)$ has no motivation. Consider, for example, a temporal sequence of information partitions with imperfect recall and suppose that the individual derives her conditional probabilistic beliefs from a full support prior. Then, considering the supports of the conditional probability distributions, one obtains a KB-frame (in particular, (R1) and (R2) will be automatically satisfied) in which condition (C) is violated. Another clear example is the simplest instance of imperfect recall, namely the case of pure loss of information, where a cell of the information partition at time $t+1$ contains the union of two or more cells of the information partition at time $t$. In this case (R2) is incompatible with (C). ${ }^{19}$

One might want to consider a weaker system than $\mathbf{K}_{\mathrm{KB}}^{\text {time }}+(\mathrm{A} 3)+(\mathrm{A} 4)$ where the truth axiom $K_{t} \phi \rightarrow \phi$ is not postulated for the knowledge operator and is replaced by the consistency axiom $\mathrm{K}_{\mathrm{t}} \phi \rightarrow \neg \mathrm{K}_{\mathrm{t}} \neg \phi$ (semantically the information function $\mathcal{K}_{\mathrm{t}}$ is serial but not necessarily reflexive). Such a system would represent situations where beliefs are still based on information, but information is not necessarily veridical, that is, the individual might receive inaccurate information and form beliefs based on it. ${ }^{20}$ In such a system, both 'knowledge' and belief would satisfy the logic of KD45. Axiom (A1) would still establish the epistemic priority of the beliefs represented by $K_{t}$ (information) over the beliefs represented by $B_{t}$, so that the receipt of new information might prompt a belief revision. Let $K_{\mathrm{KD} 45}^{\text {time }}$ denote the system obtained from $\mathrm{K}_{\mathrm{KB}}^{\mathrm{time}}$ by replacing the truth axiom for $\mathrm{K}_{\mathrm{t}}$ with the consistency axiom. Define a KD45-frame as a structure $\left\langle\Omega,\left\{\mathcal{K}_{t}\right\}_{t \in \mathbb{N}},\left\{\mathcal{B}_{t}\right\}_{t \in \mathbb{N}}\right\rangle$, where $\Omega$ is a non-empty set, $\mathcal{K}_{t}: \Omega \rightarrow 2^{\Omega}$

Proposition 5, neither (A5) nor the schema $B_{t}\left(K_{t} \phi \rightarrow K_{t+1} \phi\right)$ are theorems of $K_{K B}^{\text {time }}+(A 3)$ $+(\mathrm{A} 4)$.

${ }^{19}$ Furthermore, restricting attention (for simplicity) to the case of two dates, the following is true. Let $\mathrm{F}=\left\langle\Omega, \mathcal{K}_{0}, \mathcal{K}_{1}\right\rangle$ be such that $\mathcal{K}_{t}(t=0,1)$ is reflexive and euclidean. If $\mathrm{F}$ satisfies (R5), then, for every serial, transitive and euclidean (STE) $B_{0}$ that satisfies (R1) and (R2) there is an STE $\mathcal{B}_{1}$ that satisfies (R1), (R2) such that $\left\langle\Omega, \mathcal{K}_{0}, \mathcal{K}_{1}, \mathcal{B}_{0}, \mathcal{B}_{1}\right\rangle$ satisfies (C). On the other hand, if $F$ violates (R5) then there is an STE $B_{0}$ that satisfies (R1) and (R2) such that, for every STE $\mathcal{B}_{1}$ that satisfies (R1) and (R2), $\left\langle\Omega, \mathcal{K}_{0}, \mathcal{K}_{1}, \mathcal{B}_{0}, \mathcal{B}_{1}\right\rangle$ violates (C).

${ }^{20}$ For example, the analysis of extensive games usually involves two different epistemic levels: a player receives information which is encoded in the description of the extensive game (information sets encode information about past moves and the payoff functions encode information about preferences: this corresponds to $\mathcal{K}$ in our framework), and forms beliefs at every information set (in the form of a conditional probability distribution) concerning unobserved past moves and future moves (this corresponds to our $\mathcal{B}$ ). Although it is usually assumed that a player's information is veridical, in general it need not be. For example, a player might be mistaken about the preferences of her opponents. (In their textbook on game theory Osborne and Rubinstein, 1994, p. 135, write: 'In our view a model should attempt to capture the features of reality that the players perceive; it should not necessarily aim to describe the reality that an outside observer perceives, though obviously there are links between the two perceptions'.) 
and $\mathcal{B}_{\mathrm{t}}: \Omega \rightarrow 2^{\Omega}$ are serial, transitive and euclidean and together they satisfy (R1) and (R2). Let $\mathcal{F}_{\text {KD45 }}$ be the class of KD45-frames, $\mathcal{F}_{\mathrm{KD} 45}^{C}$ the subset of those frames that satisfy $(C)$ and $\mathbb{Q}\left(\mathcal{F}_{\mathrm{KD} 45}^{\mathrm{C}}\right)$ the class of models based on frames in $\mathcal{F}_{\mathrm{KD} 45}^{\mathrm{C}}$. The following proposition states that the conjunction of (A3) and (A4) no longer characterizes (C).

PROPOSITION 7. $\mathrm{K}_{\mathrm{KB}}^{\text {time }}+(\mathrm{A} 3)+(\mathrm{A} 4)$ is sound and complete with respect to a proper subset of $\mathbb{Q}\left(\mathcal{F}_{\mathrm{KD45}}^{\mathrm{C}}\right) .{ }^{21}$

$\mathrm{K}_{\mathrm{KB}}^{\text {time }}+(\mathrm{A} 3)+(\mathrm{A} 4)$ is complete with respect to $\mathbb{Q}\left(\mathcal{F}_{\mathrm{KD} 45}^{\mathrm{C}}\right)$ but not sound. The following example shows that soundness fails. Let $F$ be the following KD45-frame: $\Omega=\{\alpha, \beta\}, \mathcal{K}_{0}(\alpha)=\mathcal{K}_{0}(\beta)=\mathcal{B}_{0}(\alpha)=\mathcal{B}_{0}(\beta)=\{\beta\}$ and for all $t \geq 1, \mathcal{K}_{\mathrm{t}}(\alpha)=\mathcal{K}_{\mathrm{t}}(\beta)=\mathcal{B}_{\mathrm{t}}(\alpha)=\mathcal{B}_{\mathrm{t}}(\beta)=\{\alpha\}$. Then $\mathrm{F}$ satisfies $(\mathrm{C})$ (vacuously). Let $\mathscr{M}$ be a model based on $\mathrm{F}$ where $\|\pi\|=\{\beta\}$. Then at $\alpha$ both $B_{0} \pi \rightarrow B_{0} B_{1} \pi$ and $B_{0} B_{1} \neg \pi \rightarrow B_{0} \neg \pi$ are false. Hence this model does not validate (A3) and (A4).

\section{RELATED LITERATURE}

The notion of belief change has been an active area of research in philosophy and artificial intelligence. Of particular relevance to this paper is the literature that attempts to describe how an agent should accommodate a new belief (possibly inconsistent with her other beliefs) about a static world (Alchourrón, Gärdenfors and Makinson, 1985, Gärdenfors, 1988; Gä:denfors and Makinson, 1988; see also Stalnaker, 1984). In this literature an agent's beliefs are represented as a logically closed set of formulae in some language, and then constraints are put on how these beliefs can change in order to accommodate a new formula (representing new information). The main goal of this approach, as Gärdenfors (1988, p. 2) states, is to present rationality criteria for belief changes. The guiding principle is that $(1988$, p. 8) 'when evaluating changes of belief, we require that the change be the minimal one needed to accommodate the epistemic input that generates the change'. One of the results in this literature is the representation theorem for belief expansion. First we need to introduce some notation and definitions. Gärdenfors (p. 24) defines a belief set as a proper subset $\beta$ of the set of formulae such that (using our notation: cf. Definition 3) $\beta=[\beta]^{\mathrm{PL}}$. Let $\beta$ be a belief set and $\psi$ a formula such that $\neg \psi \notin \beta$. The formula $\psi$ represents an 'epistemic input', for example, a piece of information received by the individual (thus the condition $\neg \psi \notin \beta$ says that the epistemic input does not contradict her previous beliefs). The new formula $\psi$ must be accommodated in the belief set, leading to a new belief set $\beta_{\psi}^{+}$, called the expansion of $\beta$ by $\psi$. Gärdenfors (1988, pp. 48-51) gives a list of 'rationality conditions' relating to $\beta$ and $\beta_{\psi}^{+}$and proves (Theorem 3.1, p. 51) that those conditions are

${ }^{21}$ This proper subset is explicitly characterized in the proof contained in the appendix. 
satisfied if and only if (in our notation) $\beta_{\psi}^{+}=[\{\psi\} \cup \beta\}^{\mathrm{PL}}$. The close relationship between this result and Corollary 1 is apparent. However, unlike ours, Gärdenfors' approach is primarily concerned with the case in which the epistemic input contradicts the individual's prior beliefs. In this case 'revision' necessarily differs from 'expansion'. Also, Gärdenfors' work is not based on an explicit representation of time; instead potential later belief states are defined by the new information that might be received. In contrast, we index the belief states by time, leaving the content of the information that induces the change in the belief state implicit. Furthermore, Gärdenfors' analysis is not carried out within a two-level structure that explicitly distinguishes between information (evidence, knowledge) and beliefs.

Our analysis is based on a logic with two modal operators, the knowledge operator, representing information, and the belief operator. We already mentioned that the atemporal relationship between knowledge and belief was first discussed by Hintikka (1962) and Lenzen (1978). ${ }^{22}$ Kraus and Lehmann (1988) presented a formal analysis focused on soundness and completeness results. They also analyzed an atemporal multi-agent logic with operators for common knowledge and common belief. In the last part of the paper the authors considered the possibility of extending the logic to include time. In particular, they addressed the question of how to characterize the notion of persistence of beliefs: 'if person i believes something, he will keep on believing it until he knows it is false' (1988, p. 107, our emphasis). If 'something' were interpreted as the conjunction of all the propositions the individual believes, this would correspond to our property (P2) (see Proposition 3). But Kraus and Lehmann mean 'an arbitrary proposition'. In fact, they put forward an axiom schema that in our framework would be written as follows: ${ }^{23}$

$\left(\mathrm{A}^{*}\right) \mathrm{B}_{\mathrm{t}} \phi \rightarrow \mathrm{B}_{\mathrm{t}+1} \phi \vee \mathrm{K}_{\mathrm{t}+1} \neg \phi$.

$\left(\mathrm{A}^{*}\right)$ says that if at date $t$ the individual believes $\phi$, then at date $t+1$ either

22 One property of systems that model the interaction of knowledge and belief is that if one adds to (A1) and (A2) the axiom schema $\mathrm{B} \phi \rightarrow \mathrm{BK} \phi$ (if you believe something then you believe that you know it) then knowledge and belief become identical, that is, one obtains the theorem $\mathrm{B} \phi \leftrightarrow \mathrm{K} \phi$. This point was first discussed by Lenzen (1978) and later taken up by van der Hoek (1993) and Halpern (forthcoming). Van der Hoek provides an extensive analysis of the causes of this 'problem' and of a similar system that allows one to introduce the axiom $\mathrm{B} \phi \rightarrow \mathrm{BK} \phi$ without obtaining a collapse of belief into knowledge. Halpern shows that the problem can be avoided if the axiom schema $\mathrm{B} \phi \rightarrow \mathrm{BK} \phi$ is weakened so that it applies only to non-modal formulae.

23 While time enters our analysis explicitly only through the knowledge and belief operators, Kraus and Lehmann introduced a time operator $O$ in the syntax, where $O \phi$ would be interpreted as 'at the next date $\phi^{\prime}$. Thus $\left(A^{*}\right)$ below does not coincide with any of the axioms they considered. However, it can be viewed as the translation, within our framework, of their axiom (A21). 
she knows that $\phi$ is false or she still believes $\phi$. It should be clear that in non trivial models based on KB-frames $\left(A^{*}\right)$ cannot be valid. To see this, suppose that at date $t$ the individual believes that both $\phi$ and $\psi$ are true, and at date $t+1$ she learns that either $\phi$ or $\psi$ is false, but according to her new knowledge neither $\phi$ nor $\psi$ can be ruled out as false. By $\left(A^{*}\right)$ the individual should believe both $\phi$ and $\psi$ at date $t+1$, but this would contradict either (A1) (entailment axiom) or $\left(D_{B}\right)$ (consistency of beliefs). ${ }^{24}$ Besides $\left(A^{*}\right)$, Kraus and Lehmann briefly discussed a number of possible axioms (all of them different from our (A3) and (A4)) and concluded by saying that 'An open problem is: find a natural family of models for which the systems considered above are complete' ${ }^{25}$

Some of the papers mentioned above deal with the more general case where there are $n \geq 1$ individuals, whereas we have restricted attention to the case of one individual. It should be clear, however, that our approach can be extended to the multi-agent case in order to analyze dynamic, interactive epistemic systems.

\section{APPENDIX}

Proof of Proposition 1. Fix an arbitrary frame $\mathrm{F} \in \mathcal{F}_{\mathrm{KB}}$. First we show that $(\mathrm{P} 1)$ is equivalent to the following:

(R3) $\forall \omega, \omega^{\prime} \in \Omega, \forall t \in \mathbb{N}$, if $\omega^{\prime} \in \mathcal{B}_{\mathrm{t}}(\omega)$ then there exists an $\omega^{\prime \prime} \in \mathcal{B}_{\mathrm{t}}(\omega)$ such that $\omega^{\prime} \in \mathcal{B}_{\mathrm{t}+1}\left(\omega^{\prime \prime}\right)$.

$(\mathrm{R} 3) \Rightarrow(\mathrm{P} 1)$. Fix arbitrary $t, \omega$ and $\omega^{\prime}$ such that $\omega^{\prime} \in \mathcal{B}_{\mathrm{t}}(\omega) \cap \mathcal{K}_{\mathrm{t}+1}(\omega)$. We want to show that $\omega^{\prime} \in \mathcal{B}_{\mathrm{t}+1}(\omega)$. By (R3) there exists an $\omega^{\prime \prime} \in \mathcal{B}_{\mathrm{t}}(\omega)$ such that $\omega^{\prime} \in \mathcal{B}_{t+1}\left(\omega^{\prime \prime}\right)$. Since $\mathcal{B}_{t+1}$ is euclidean, $\omega^{\prime} \in \mathcal{B}_{t+1}\left(\omega^{\prime}\right)$. By $(R 2)$, since $\omega^{\prime} \in \mathcal{K}_{\mathrm{t}+1}(\omega), \mathcal{B}_{\mathrm{t}+1}\left(\omega^{\prime}\right) \subseteq \mathcal{B}_{\mathrm{t}+1}(\omega)$. Hence $\omega^{\prime} \in \mathcal{B}_{\mathrm{t}+1}(\omega)$.

$(\mathrm{P} 1) \Rightarrow(\mathrm{R} 3)$. Fix arbitrary $t, \omega$ and $\omega^{\prime}$ such that $\omega^{\prime} \in \mathcal{B}_{\mathrm{t}}(\omega)$. We want to show that there exists an $\omega^{\prime \prime} \in \mathcal{B}_{\mathrm{t}}(\omega)$ such that $\omega^{\prime} \in \mathcal{B}_{\mathrm{t}+1}\left(\omega^{\prime \prime}\right)$. Take $\omega^{\prime \prime}=\omega^{\prime}$. Then we only have to show that $\omega^{\prime} \in \mathcal{B}_{t+1}\left(\omega^{\prime}\right)$. By reflexivity of

24 For example, consider a model where: $\Omega=\{\alpha, \beta, \gamma\}, \forall \omega \in \Omega, \mathcal{K}_{0}(\omega)=\Omega, \mathcal{B}_{0}(\omega)=\{\alpha\}$, for all $\mathrm{t} \geq 1, \mathcal{K}_{\mathrm{t}}(\alpha)=\{\alpha\}, \mathcal{K}_{\mathrm{t}}(\beta)=\mathcal{K}_{\mathrm{t}}(\gamma)=\mathcal{B}_{\mathrm{t}}(\beta)=\mathcal{B}_{\mathrm{t}}(\gamma)=\{\beta, \gamma\},\|\phi\|=\{\alpha, \beta\},\|\psi\|=\{\alpha, \gamma\}$. Then $B_{0} \phi, B_{0} \psi, \neg K_{1} \neg \phi, \neg K_{1} \neg \psi$ and $K_{1} \neg(\phi \wedge \psi)$ hold at $\beta$ and $\gamma$. Thus (A1) implies that $B_{1} \neg(\phi \wedge \psi)$ holds at $\beta$ and $\gamma$, while $\left(A^{*}\right)$ would imply that $B_{1}(\phi \wedge \psi)$ holds at $\beta$ and $\gamma$.

25 Van der Hoek and Meyer (1995, p. 96) review the axioms proposed by Kraus and Lehmann (1988) and state 'As fas as is known to us, it is still an open problem to find a natural family of models with respect to which these systems are complete'. In a very recent paper Friedman and Halpern (1995) provide a general semantic analysis of belief change in terms of knowledge and plausibility, the latter being a generalization of probabilistic beliefs (an agent believes $\phi$ if he knows that $\neg \phi$ is implausible). In the last section they introduce time into their analysis and give an axiom (which they call $\mathrm{COH}$, for coherence) which is somewhat related to our axioms (A3) and (A4). However, in what would be their analogue of our framework, the system containing axiom $\mathrm{COH}$ is sound but not complete (Halpern, private communication). 
$\mathcal{K}_{\mathrm{t}+1}, \omega^{\prime} \in \mathcal{K}_{\mathrm{t}+1}\left(\omega^{\prime}\right)$. By euclideanness of $\mathcal{B}_{\mathrm{t}}$, since $\omega^{\prime} \in \mathcal{B}_{\mathrm{t}}(\omega), \omega^{\prime} \in \mathcal{B}_{\mathrm{t}}\left(\omega^{\prime}\right)$. By $(\mathrm{P} 1)$, since $\omega^{\prime} \in \mathcal{B}_{\mathrm{t}}\left(\omega^{\prime}\right) \cap \mathcal{K}_{\mathrm{t}+1}\left(\omega^{\prime}\right), \omega^{\prime} \in \mathcal{B}_{\mathrm{t}+1}\left(\omega^{\prime}\right)$.

Next we show that (P2) is equivalent to the following:

(R4) $\forall \omega, \omega^{\prime}, \omega^{\prime \prime} \in \Omega, \forall t \in \mathbb{N}$, if $\omega^{\prime} \in \mathcal{B}_{\mathrm{t}}(\omega)$ and $\omega^{\prime \prime} \in \mathcal{B}_{\mathrm{t}+1}\left(\omega^{\prime}\right)$ then $\omega^{\prime \prime} \in \mathcal{B}_{\mathrm{l}}(\omega)$.

(R4) $\Rightarrow(\mathrm{P} 2)$. Fix arbitrary $t, \omega$ and $\omega^{\prime}$ such that $\omega^{\prime} \in \mathcal{B}_{t}(\omega) \cap \mathcal{K}_{\mathrm{t}+1}(\omega)$ (so that $\mathcal{B}_{\mathrm{t}}(\omega) \cap \mathcal{K}_{\mathrm{t}+1}(\omega) \neq \emptyset$ ). We want to show that $\mathcal{B}_{\mathrm{t}+1}(\omega) \subseteq \mathcal{B}_{\mathrm{t}}(\omega)$. Fix an $\hat{\omega} \in \mathcal{B}_{\mathrm{t}+1}\left(\omega^{\prime}\right)$ (it exists because $\mathcal{B}_{\mathrm{t}+1}$ is serial). By (R4), since $\omega^{\prime} \in \mathcal{B}_{\mathrm{t}}(\omega)$ and $\hat{\omega} \in \mathcal{B}_{\mathrm{t}+1}\left(\omega^{\prime}\right), \hat{\omega} \in \mathcal{B}_{\mathrm{t}}(\omega)$. By $(\mathrm{R} 2)$, since $\omega^{\prime} \in \mathcal{K}_{\mathrm{t}+1}(\omega), \mathcal{B}_{\mathrm{t}+1}\left(\omega^{\prime}\right) \subseteq \mathcal{B}_{\mathrm{t}+1}(\omega)$. Hence $\hat{\omega} \in \mathcal{B}_{\mathrm{t}+1}(\omega)$. It follows from euclideanness of $\mathcal{B}_{\mathrm{t}+1}$ that $\mathcal{B}_{\mathrm{t}+1}(\omega) \subseteq$ $\mathcal{B}_{t+1}(\hat{\omega})$. Now choose an arbitrary $\omega^{\prime \prime} \in \mathcal{B}_{t+1}(\omega)$. Then $\omega^{\prime \prime} \in \mathcal{B}_{t+1}(\hat{\omega})$. By (R4), since $\hat{\omega} \in \mathcal{B}_{\mathrm{t}}(\omega)$ and $\omega^{\prime \prime} \in \mathcal{B}_{\mathrm{t}+1}(\hat{\omega}), \omega^{\prime \prime} \in \mathcal{B}_{\mathrm{t}}(\omega)$. Thus $\mathcal{B}_{\mathrm{t}+1}(\omega) \subseteq \mathcal{B}_{\mathrm{t}}(\omega)$.

(P2) $\Rightarrow$ (R4). Fix arbitrary $t, \omega, \omega^{\prime}$ and $\omega^{\prime \prime}$ such that $\omega^{\prime} \in \mathcal{B}_{\mathrm{t}}(\omega)$ and $\omega^{\prime \prime} \in \mathcal{B}_{\mathrm{t}+1}\left(\omega^{\prime}\right)$. We want to show that $\omega^{\prime \prime} \in \mathcal{B}_{\mathrm{t}}(\omega)$. By reflexivity of $\mathcal{K}_{\mathrm{t}+1}$, $\omega^{\prime} \in \mathcal{K}_{t+1}\left(\omega^{\prime}\right)$. Since $\omega^{\prime} \in \mathcal{B}_{\mathrm{t}}(\omega)$, by euclideanness and transitivity of $\mathcal{B}_{\mathrm{t}}$, $\mathcal{B}_{\mathrm{t}}(\omega)=\mathcal{B}_{\mathrm{t}}\left(\omega^{\prime}\right)$. Thus $\omega^{\prime} \in \mathcal{B}_{\mathrm{t}}\left(\omega^{\prime}\right)$. By $(\mathrm{P} 2)$, since $\omega^{\prime} \in \mathcal{B}_{\mathrm{t}}\left(\omega^{\prime}\right) \cap \mathcal{K}_{\mathrm{t}+1}\left(\omega^{\prime}\right)$, $\mathcal{B}_{\mathrm{t}+1}\left(\omega^{\prime}\right) \subseteq \mathcal{B}_{\mathrm{t}}\left(\omega^{\prime}\right)$. Hence $\omega^{\prime \prime} \in \mathcal{B}_{\mathrm{t}}(\omega)=\mathcal{B}_{\mathrm{t}}\left(\omega^{\prime}\right)$.

To complete the proof we invoke Theorem 4.3 in van der Hoek (1993, p.183), which, adapted to our framework, states that a frame satisfies (R3) if and only if for every model $\mathscr{M}$ based on it and for all $t$ and $\phi$, the formula $\left(B_{t} B_{t+1} \phi \rightarrow B_{t} \phi\right)$ is valid in $\mathscr{M}$ and it satisfies (R4) if and only if for every model $\mathscr{M}$ based on it and for all $t$ and $\phi$, the formula $\left(\mathrm{B}_{\mathrm{t}} \phi \rightarrow \mathrm{B}_{\mathrm{t}}\right.$ $\left.\mathrm{B}_{\mathrm{t}+1} \phi\right)$ is valid in $\mathscr{M}$.

Proof of Proposition 2. (1) $\Rightarrow$ (2). Let F be a frame that satisfies (P1). Fix an arbitrary model $\mathscr{M} \in \mathbb{Q}(\mathrm{F})$ and arbitrary $t \in \mathbb{N}, \omega \in \Omega$ and $\phi \in \beta$ $(t+1, \omega)$ (to simplify the notation we omit the superscript $\mathscr{M}$ ). Then $\models_{\omega} B_{t+1} \phi$. By (R2) (cf. Remark 1) $\models_{\omega}\left(B_{t+1} \phi \rightarrow K_{t+1} B_{t+1} \phi\right)$. Hence $\models_{\omega} K_{t+1}$ $\mathrm{B}_{\mathrm{t}+1} \phi$. Thus

$$
\mathrm{B}_{\mathrm{t}+1} \phi \in \kappa(t+1, \omega) \text {. }
$$

By euclideanness of $\mathcal{B}_{t+1}$, for every $\alpha \in \Omega, \vDash_{\alpha} B_{t+1}\left(B_{t+1} \phi \rightarrow \phi\right)$ (it is a theorem of the logic $K 5$ (see Section 3 ), hence valid in every euclidean model). Thus $F_{\omega} B_{t} B_{t+1}\left(B_{t+1} \phi \rightarrow \phi\right)$. Since $F$ satisfies (P1), by (1) of Proposition $1, F_{\omega} B_{t} B_{t+1}\left(B_{t+1} \phi \rightarrow \phi\right) \rightarrow B_{t}\left(B_{t+1} \phi \rightarrow \phi\right)$. Thus $\vDash_{\omega} B_{t}\left(B_{t+1}\right.$ $\phi \rightarrow \phi)$, that is,

$$
\left(B_{t+1} \phi \rightarrow \phi\right) \in \beta(t, \omega) .
$$

Hence, by (a.1) and (a.2), (2) is satisfied with $\psi=B_{t+1} \phi$.

Not (1) $\Rightarrow$ not (2). Suppose that $F$ does not satisfy (P1). Then there exist $t$ and $\omega$ such that $\mathcal{B}_{\mathrm{t}}(\omega) \cap \mathcal{K}_{\mathrm{t}+1}(\omega) \nsubseteq \mathcal{B}_{\mathrm{t}+1}(\omega)$, that is, there exists an $\alpha \in \mathcal{B}_{\mathfrak{t}}(\omega) \cap \mathcal{K}_{t+1}(\omega)$ such that $\alpha \notin \mathcal{B}_{t+1}(\omega)$. Let $\pi$ be a sentence letter and $\mathscr{M} \in \mathbb{Q}(\mathrm{F})$ be a model where $\|\pi\|=\mathcal{B}_{t+1}(\omega)$. Then $\pi \in \beta(t+1, \omega)$. Choose 
an arbitrary $\psi \in \kappa(t+1, \omega)$. Then $\mathcal{K}_{t+1}(\omega) \subseteq\|\psi\|$. Since $\alpha \in \mathcal{B}_{t}(\omega)$ and $\alpha \in \mathcal{K}_{t+1}(\omega) \cap-\mathcal{B}_{t+1}(\omega) \subseteq\|\psi\| \cap\|\neg \pi\|=\|\psi \wedge \neg \pi\|=\|\neg(\psi \rightarrow \pi)\|, \quad(\psi \rightarrow \pi)$ $\notin \beta(t, \omega)$, that is, $(2)$ is violated.

Remark A.1. The proof of Proposition 2 does not make use of reflexivity of $\mathcal{K}_{t}$ nor of transitivity of $\mathcal{B}_{t}$ and $\mathcal{K}_{t}$. Thus Proposition 2 holds in a larger class than $\mathcal{F}_{\text {KB. }}$

To prove Proposition 3 we need the following Lemma.

Lemma A.1. Fix an arbitrary frame $F \in \mathcal{F}_{\mathrm{KB}}$ and arbitrary $t \in \mathbb{N}$ and $\omega \in \Omega$. Then the following are equivalent:

(1) $\mathcal{B}_{\mathrm{t}}(\omega) \cap \mathcal{K}_{\mathrm{t}+1}(\omega) \neq \emptyset$,

(2) for every $\mathscr{M} \in \mathbb{M}(\mathrm{F}), \beta^{\mathscr{M}}(t, \omega) \cup \kappa^{\mathscr{H}}(t, \omega)$ is consistent.

Proof. Not (2) $\Rightarrow$ not (1). Let $\mathscr{M} \in \mathbb{Q}(\mathrm{F})$ be a model where $\beta(t, \omega) \cup$ $\kappa(t+1, \omega)$ is inconsistent. Then, since both $\beta(t, \omega)$ and $\kappa(t, \omega)$ are consistent, there must be a formula $\phi$ such that $\phi \in \beta(t, \omega)$ and $\neg \phi \in \kappa(t, \omega)$, that is, $\mathcal{B}_{t}(\omega) \subseteq\|\phi\|$ and $\mathcal{K}_{t+1}(\omega) \subseteq\|\neg \phi\|=-\|\phi\|$. Then $\mathcal{B}_{\mathrm{t}}(\omega) \cap \mathcal{K}_{\mathrm{t}+1}(\omega) \subseteq\|\phi\| \cap-\|\phi\|=\emptyset$.

Not (1) $\Rightarrow$ not (2). Suppose that $\mathcal{B}_{\mathrm{t}}(\omega) \cap \mathcal{K}_{\mathrm{t}+1}(\omega)=\varnothing$, that is, $\mathcal{K}_{\mathrm{t}+1}(\omega)$ $\subseteq-\mathcal{B}_{\mathrm{t}}(\omega)$. Let $\pi$ be a sentence letter and $\mathscr{M} \in \mathbb{Q}(\mathrm{F})$ a model where $\|\pi\|=$ $\mathcal{B}_{\mathrm{t}}(\omega)$. Then $\pi \in \beta(t, \omega)$ and $\mathcal{K}_{\mathrm{t}+1}(\omega) \subseteq\|\neg \pi\|$, that is, $\neg \pi \in \kappa(t+1, \omega)$ and, therefore, $\beta(t, \omega) \cup \kappa(t+1, \omega)$ is inconsistent.

Proof of Proposition 3. (1) $\Rightarrow$ (2). Let $\mathrm{F} \in \mathcal{F}_{\mathrm{KB}}$ be a frame that satisfies (P2). Fix arbitrary $\mathscr{M} \in \mathbb{M}(\mathrm{F}), t \in \mathbb{N}$ and $\omega \in \Omega$ such that $\beta(t, \omega) \cup \kappa(t+1, \omega)$ is consistent (once again we drop the superscript $\mathscr{M}$ ). Choose an arbitrary $\phi \in \beta(t, \omega)$, i.e. $\models_{\omega} B_{t} \phi$. By (2) of Proposition $1, \vDash_{\omega} B_{t} \phi \rightarrow B_{t} B_{t+1} \phi$. Hence $F_{\omega} B_{t} B_{t+1} \phi$, that is, $B_{t+1} \phi \in \beta(t, \omega)$. Since $\beta(t, \omega) \cup \kappa(t+1, \omega)$ is consistent, $\neg B_{t+1} \phi \notin \kappa(t+1, \omega)$ i.e. $\mathcal{K}_{t+1}(\omega) \nsubseteq-\left\|B_{t+1} \phi\right\|$. Thus there exists an $\alpha \in \mathcal{K}_{t+1}(\omega)$ such that $\models_{\alpha} B_{t+1} \phi$. By (R2) (cf. Remark 1) $\models_{\alpha} B_{t+1} \phi \rightarrow K_{t+1}$ $\mathrm{B}_{t+1} \phi$. Hence $\vDash_{\alpha} K_{t+1} B_{t+1} \phi$ and, therefore, $\models_{\omega} \neg K_{t+1} \neg K_{t+1} B_{t+1} \phi$. Since $\mathcal{K}_{t+1}$ is euclidean, $\models_{\omega} \neg K_{t+1} \neg K_{t+1} B_{t+1} \phi \rightarrow K_{t+1} B_{t+1} \phi$. Hence $\models_{\omega} K_{t+1} B_{t+1} \phi$. By (R1) (cf. Remark 1) $\models_{\omega} K_{t+1} B_{t+1} \phi \rightarrow B_{t+1} B_{t+1} \phi$. Thus $\models_{\omega} B_{t+1} B_{t+1} \phi$. By euclideanness of $B_{t+1}, \models_{\omega} B_{t+1} B_{t+1} \phi \rightarrow B_{t+1} \phi$ (it is a theorem of the logic K5 (see Section 3) hence valid in every euclidean frame). Thus $\models_{\omega} B_{t+1} \phi$, that is, $\phi \in \beta(t+1, \omega)$. Since $\phi \in \beta(t, \omega)$ was chosen arbitrarily, $\beta(t, \omega) \subseteq \beta(t+1, \omega)$.

not $(1) \Rightarrow \operatorname{not}(2)$. Let $F \in \mathcal{F}_{\mathrm{KB}}$ be a frame that violates (P2). Then there exist $t$ and $\omega$ such that $\mathcal{B}_{\mathrm{t}}(\omega) \cap \mathcal{K}_{\mathrm{t}+1}(\omega) \neq \emptyset$ and $\mathcal{B}_{\mathrm{t}+1}(\omega) \nsubseteq \mathcal{B}_{\mathrm{t}}(\omega)$, that is, there exists an $\alpha \in \mathcal{B}_{\mathrm{t}+1}(\omega)$ such that $\alpha \notin \mathcal{B}_{\mathrm{t}}(\omega)$. Let $\pi$ be a sentence letter and $\mathscr{M} \in \mathbb{Q}(\mathrm{F})$ be a model where $\|\pi\|=\mathcal{B}_{\mathrm{t}}(\omega)$. Thus $\pi \in \beta(t, \omega)$. Since $\mathcal{B}_{\mathrm{t}}(\omega) \cap \mathcal{K}_{\mathrm{t}+1}(\omega) \neq \varnothing$, by Lemma A.1 $\beta(t, \omega) \cup \kappa(t+1, \omega)$ is consistent. 
Furthermore, $\pi \notin \beta(t+1, \omega)$ since $\alpha \in \mathcal{B}_{\mathrm{t}+1}(\omega)$ and $\alpha \in-\mathcal{B}_{\mathrm{t}}(\omega)=\|\neg \pi\|$. Thus $\beta(t, \omega) \nsubseteq \beta(t+1, \omega)$.

Remark A.2. The proof of Proposition 3 does not make use of reflexivity of $\mathcal{K}_{\mathrm{t}}$ nor of transitivity of $\mathcal{B}_{\mathrm{t}}$ and $\mathcal{K}_{\mathrm{t}}$. Thus Proposition 3 holds in a larger class than $\mathcal{F}_{\mathrm{KB}}$.

In order to prove Corollary 1 we need the following lemmas.

Lemma A.2. Fix arbitrary $\mathrm{F} \in \mathcal{F}_{\mathrm{KB}}, t \in \mathbb{N}, \omega \in \Omega$ and $\mathscr{M} \in \mathbb{Q}(\mathrm{F})$. Then the following are equivalent:

(1) if $\phi \in \beta^{\mathscr{M}}(t+1, \omega)$ then $\exists \psi \in \kappa^{\mathscr{M}}(t+1, \omega)$ such that $(\psi \rightarrow \phi) \in$ $\beta^{\mathscr{H}}(t, \omega)$,

(2) $\beta^{\mathscr{\mu}}(t+1, \omega) \subseteq\left[\beta^{\mathscr{\mu}}(t, \omega) \cup \kappa^{\mathscr{N}}(t+1, \omega)\right]^{\mathrm{PL}}$,

(3) if $\beta^{\mathscr{H}}(t, \omega) \cup \kappa^{\mathscr{N}}(t+1, \omega)$ is consistent then $\beta^{\mathscr{\mu}}(t+1, \omega) \subseteq$ $\left[\beta^{\mathscr{\mu}}(t, \omega) \cup \kappa^{\mathscr{M}}(t+1, \omega)\right]^{\mathrm{PL}}$.

Proof. (1) $\Rightarrow$ (2). Choose an arbitrary $\phi \in \beta^{\mathscr{A}}(t+1, \omega)$. By (1) $\exists \psi \in$ $\kappa^{\mathscr{\mu}}(t+1, \omega)$ such that $(\psi \rightarrow \phi) \in \beta^{\mu \prime}(t, \omega)$. Since $\psi,(\psi \rightarrow \phi) \in \beta^{\mu \prime}(t, \omega) \cup$ $\kappa^{\mathscr{M}}(t+1, \omega)$ and $(\psi \wedge(\psi \rightarrow \phi)) \rightarrow \phi$ is a tautology, $\phi \in\left[\beta^{\mathscr{H}}(t, \omega) \cup\right.$ $\left.\kappa^{\mathscr{\mu}}(t+1, \omega)\right]^{\mathrm{PL}}$.

(2) $\Rightarrow$ (1). Fix an arbitrary $\phi \in \beta^{\mu \prime}(t+1, \omega)$. By (2) $\phi \in\left[\beta^{\mu}(t, \omega) \cup\right.$ $\left.\kappa^{\mathscr{M}}(t+1, \omega)\right]^{\mathrm{PL}}$, that is, there exist $\phi_{1}, \ldots, \phi_{n} \in \beta^{\mathcal{H}}(t, \omega) \cup \kappa^{\mathscr{\mu}}(t+1, \omega)$ such that $\left(\phi_{1} \wedge \ldots \wedge \phi_{n}\right) \rightarrow \phi$ is a tautology. If $\phi_{i} \in \kappa^{\mu}(t+1, \omega)$ for all $\mathrm{i}=1, \ldots, \mathrm{n}$, then $\left(\phi_{1} \wedge \ldots \wedge \phi_{\mathrm{n}}\right) \in \kappa^{\mu}(t+1, \omega)$ (see Chellas, 1984) and, therefore, (1) holds with $\psi=\left(\phi_{1} \wedge \ldots \wedge \phi_{n}\right)$. If $\phi_{i} \in \beta^{\mathcal{M}}(t, \omega)$ for all $\mathrm{i}=1, \ldots, \mathrm{n}$, then $\left(\phi_{1} \wedge \ldots \wedge \phi_{\mathrm{n}}\right) \in \beta^{\mathcal{M}}(t, \omega)$. It follows that $\phi \in \beta^{\mathcal{M}}(t, \omega)$ and therefore (1) holds for arbitrary $\psi \in \kappa^{\mu}(t+1, \omega)$. Finally, let $1 \leq k<n$ be such that (after a renumbering of the formulae, if necessary) $\phi_{1}, \ldots, \phi_{\mathrm{k}}$ $\in \beta^{\mathscr{\mu}}(t, \omega)$ and $\phi_{k}, \ldots, \phi_{n} \in \kappa^{\mathscr{\mu}}(t+1, \omega)$. Let $\chi=\left(\phi_{1} \wedge \ldots \wedge \phi_{k}\right)$ and $\psi=\left(\phi_{k} \wedge \ldots \wedge \phi_{n}\right)$. Then $\chi \in \beta^{\mathscr{\mu}}(t, \omega)$ and $\psi \in \kappa^{\mathscr{M}}(t+1, \omega)$. Since, by hypothesis, $(\chi \wedge \psi) \rightarrow \phi$ is a tautology and it is equivalent to $\chi \rightarrow(\psi \rightarrow \phi)$, the latter is a tautology and hence belongs to $\beta^{\mathcal{H}}(t, \omega)$. Hence $(\psi \rightarrow \phi) \in \beta^{\mathscr{H}}(t, \omega)$.

(2) $\Leftrightarrow(3)$. That (2) implies (3) is obvious. That (3) implies (2) follows from the fact that if $\beta^{\mathscr{M}}(t, \omega) \cup \kappa^{\mathscr{H}}(t+1, \omega)$ is inconsistent, then there is a formula $\phi$ such that both $\phi$ and $\neg \phi$ belong to $\beta^{\mathscr{\mu}}(t, \omega) \cup \kappa^{\mathscr{\mu}}(t+1, \omega)$ and, therefore, since, for every formula $\psi,(\phi \wedge \neg \phi) \rightarrow \psi$ is a tautology, $\left[\beta^{\mathscr{M}}(t, \omega) \cup \kappa^{\mathscr{H}}(t+1, \omega)\right]^{\mathrm{PL}}=\Phi \supseteq \beta^{\mathscr{M}}(t+1, \omega)$.

Lemma A.3. Fix arbitrary $\mathrm{F} \in \mathcal{F}_{\mathrm{KB}}, t \in \mathbb{N}, \omega \in \Omega$ and $\mathscr{M} \in \mathbb{M}(\mathrm{F})$. Then the following are equivalent:

(1) $\beta^{\mathscr{H}}(t, \omega) \subseteq \beta^{\mathscr{\mu}}(t+1, \omega)$,

(2) $\left[\beta^{\mathscr{\mu}}(t, \omega) \cup \kappa^{\mathscr{M}}(t+1, \omega)\right]^{\mathrm{PL}} \subseteq \beta^{\mathscr{\mu}}(t+1, \omega)$. 
Proof. (1) $\Rightarrow(2)$. Assume (1). By (R1) (cf. Remark 1), $\kappa^{\mu /}(t+1, \omega) \subseteq$ $\beta^{\mathscr{\mu}}(t+1, \omega)$. Hence $\beta^{\mathscr{M}}(t, \omega) \cup \kappa^{\mathscr{M}}(t+1, \omega) \subseteq \beta^{\mathscr{\mu}}(t+1, \omega)$ and, therefore, $\left[\beta^{\mathscr{A}}(t, \omega) \cup \kappa^{\mathscr{M}}(t+1, \omega)\right]^{\mathrm{PL}} \subseteq\left[\beta^{\mathscr{A}}(t+1, \omega)\right]^{\mathrm{PL}}=\beta^{\mathscr{A}}(t+1, \omega)$.

$(2) \Rightarrow(1)$. Assume (2). Fix an arbitrary $\phi \in \beta^{\mathscr{H}}(t, \omega)$. Since $\phi \rightarrow \phi$ is a tautology, $\phi \in\left[\beta^{\mathscr{H}}(t, \omega) \cup \kappa^{\prime \prime}(t+1, \omega)\right]^{\mathrm{PL}}$. Hence $\phi \in \beta^{\mathscr{N}}(t+1, \omega)$.

Proof of Corollary 1. Let $\mathrm{F} \in \mathcal{F}_{\mathrm{KB}}$. $\mathrm{F}$ satisfies (C) if and only if it satisfies (P1) and (P2). By Proposition 2 and Lemma A.2, F satisfies (P1) if and only if $\forall \mathscr{M} \in \mathbb{M}(\mathrm{F}), \forall t \in \mathbb{N}, \forall \omega \in \Omega$, if $\dot{\beta}^{\mathscr{\prime}}(t, \omega) \cup \kappa^{\mathscr{M}}(t+1, \omega)$ is consistent then $\left[\beta^{\mathscr{M}}(t, \omega) \cup \kappa^{\mathscr{N}}(t+1, \omega]^{\mathrm{PL}} \supseteq \beta^{\mathscr{M}}(t+1, \omega)\right.$. By Proposition 3 and Lemma A.3, F satisfies (P2) if and only if $\forall \mathscr{M} \in \mathbb{W}(\mathrm{F}), \forall t \in \mathbb{N}, \forall \omega \in \Omega$, if $\beta^{\mathscr{A}}(t, \omega) \cup \kappa^{\mathscr{M}}(t+1, \omega)$ is consistent then $\left[\beta^{\mathscr{\prime}}(t, \omega) \cup \kappa^{\mathscr{M}}(t+1, \omega)\right]^{\mathrm{PL}} \subseteq$ $\beta^{\prime \prime}(t+1, \omega)$.

Proof of Proposition 4. We prove by induction that if

$$
\mathrm{B}_{\mathrm{t}} \phi \leftrightarrow \mathrm{B}_{\mathrm{t}} \mathrm{B}_{\mathrm{t}+\mathrm{k}} \phi
$$

is a theorem of $\mathrm{K}_{\mathrm{KB}}^{\text {time }}+(\mathrm{A} 3)+(\mathrm{A} 4)$ (it clearly is for $\mathrm{k}=1$ ) then so is $\mathrm{B}_{\mathrm{t}} \phi \leftrightarrow \mathrm{B}_{\mathrm{t}} \mathrm{B}_{\mathrm{t}+\mathrm{k}+1} \phi$.
1. $\quad B_{t} \phi \leftrightarrow B_{t} B_{t+k} \phi$
(hypothesis)
2. $\mathrm{B}_{\mathrm{t}+\mathrm{k}} \phi \leftrightarrow \mathrm{B}_{\mathrm{t}+\mathrm{k}} \mathrm{B}_{\mathrm{t}+\mathrm{k}+1} \phi$
((A3) and (A4))
3. $B_{t} B_{t+k} \phi \leftrightarrow B_{t} B_{t+k} B_{t+k+1} \phi$
(2, RE: see Chellas, 1984, p. 17)
4. $\quad B_{t} \phi \leftrightarrow B_{t} B_{t+k} B_{t+k+1} \phi$
(1, 3, Propositional Logic)
5. $B_{t} B_{t+k+1} \phi \leftrightarrow B_{t} B_{t+k} B_{t+k+1} \phi$
(hypothesis applied to the formula
$\left.B_{t+k+1} \phi\right)$
6. $\quad B_{t} \phi \leftrightarrow B_{t} B_{t+k+1} \phi$
(4, 5, Propositional Logic).

Proof of Proposition 5. Let $\mathcal{F}_{\mathrm{KB}}^{\mathrm{R} 3-\mathrm{R} 4}$ be the class of KB-frames that satisfy properties (R3) and (R4) given in the proof of Proposition 1 and let $\mathbb{Q}\left(\mathcal{F}_{\mathrm{KB}}^{\mathrm{RB}} \mathrm{R4}\right)$ be the class of models based on frames in $\mathcal{F}_{\mathrm{KB}}^{\mathrm{R} 3-\mathrm{R} 4}$. It follows from Theorem 4.3 in van der Hoek (1993, p. 183) that $K_{\mathbf{K B}}^{\text {time }}+(\mathrm{A3})+(\mathrm{A} 4)$ is sound and complete with respect to $\mathbb{Q}\left(\mathcal{F}_{\mathrm{KB}}^{\mathrm{R} 3-\mathrm{R} 4}\right)$. By Proposition 1, a KB-frame satisfies (R3) and (R4) if and only if it satisfies (P1) and (P2). Since in every KB-frame the conjunction of (P1) and (P2) is equivalent to (C), $\mathcal{F}_{\mathrm{KB}}^{\mathrm{R} 3-\mathrm{R} 4}=\mathcal{F}_{\mathrm{KB}}^{\mathrm{C}}$.

Proof of Proposition 6. First we show that (A6) is a theorem of $\mathbf{K}_{\mathbf{K B}}^{\text {time }}+(\mathrm{A} 5)$ :

$$
\text { 1. } \neg \mathrm{K}_{\mathrm{t}} \phi \rightarrow \mathrm{K}_{\mathrm{t}} \neg \mathrm{K}_{\mathrm{t}} \phi \quad \text { (axiom } 5_{\mathrm{K}} \text { ) }
$$



2. $\quad \mathrm{K}_{\mathrm{t}} \neg \mathrm{K}_{\mathrm{t}} \phi \rightarrow \mathrm{K}_{\mathrm{t}+1} \neg \mathrm{K}_{\mathrm{t}} \phi$
(instance of (A5))
3. $\neg \mathrm{K}_{\mathrm{t}} \phi \rightarrow \mathrm{K}_{\mathrm{t}+1} \neg \mathrm{K}_{\mathrm{t}} \phi$
(1,2, Propositional Logic).

Next we show that $(A 7)$ is a theorem of $K_{K_{B}}^{\text {time }}+(A 6)$ :

1. $\neg \mathrm{K}_{\mathrm{t}} \phi \rightarrow \mathrm{K}_{\mathrm{t}+1} \neg \mathrm{K}_{\mathrm{t}} \phi$ (axiom (A6))

2. $\neg \mathrm{K}_{\mathrm{t}+1} \neg \mathrm{K}_{\mathrm{t}} \phi \rightarrow \mathrm{K}_{\mathrm{t}} \phi$ (1, Propositional Logic)

3. $\mathrm{K}_{\mathrm{t}+1} \neg \mathrm{K}_{\mathrm{t}+1} \neg \mathrm{K}_{\mathrm{t}} \phi \rightarrow \mathrm{K}_{\mathrm{t}+1} \mathrm{~K}_{\mathrm{t}} \phi$ (2, rule RK for $\mathrm{K}_{\mathrm{t}+1}$ : see Chellas, 1984, p. 19)

4. $\neg \mathrm{K}_{\mathrm{t}+1} \neg \mathrm{K}_{\mathrm{t}} \phi \rightarrow \mathrm{K}_{\mathrm{t}+1} \neg \mathrm{K}_{\mathrm{t}+1} \neg \mathrm{K}_{\mathrm{t}} \phi$ (axiom $5_{\mathrm{K}}$ applied to the formula $\left.\neg \mathrm{K}_{\mathrm{t}} \phi\right)$

5. $\neg \mathrm{K}_{\mathrm{t}+1} \neg \mathrm{K}_{\mathrm{t}+1} \neg \mathrm{K}_{\mathrm{t}} \phi \rightarrow \mathrm{K}_{\mathrm{t}+1} \neg \mathrm{K}_{\mathrm{t}} \phi$ (4, Propositional Logic)

6. $\mathrm{K}_{\mathrm{t}+1} \neg \mathrm{K}_{\mathrm{t}} \phi \rightarrow \neg \mathrm{K}_{\mathrm{t}} \phi$ (axiom $T_{K}$ applied to the formula $\left.\neg \mathrm{K}_{\mathrm{t}} \phi\right)$

7. $\neg \mathrm{K}_{\mathrm{t}+1} \neg \mathrm{K}_{\mathrm{t}+1} \neg \mathrm{K}_{\mathrm{t}} \phi \rightarrow \neg \mathrm{K}_{\mathrm{t}} \phi \quad$ (5, 6, Propositional Logic)

8. $\mathrm{K}_{\mathrm{t}} \phi \rightarrow \mathrm{K}_{\mathrm{t}+1} \neg \mathrm{K}_{\mathrm{t}+1} \neg \mathrm{K}_{\mathrm{t}} \phi \quad$ (7, Propositional Logic)

9. $\mathrm{K}_{\mathrm{t}} \phi \rightarrow \mathrm{K}_{\mathrm{t}+1} \mathrm{~K}_{\mathrm{t}} \phi$ (8, 3, Propositional Logic).

Next we show that (A5) is a theorem of $K_{K B}^{\text {time }}+(A 7)$ :

1. $\mathrm{K}_{\mathrm{t}} \phi \rightarrow \phi \quad$ (axiom $\mathrm{T}$ for $\mathrm{K}_{\mathrm{t}}$ )

2. $\mathrm{K}_{\mathrm{t}+1} \mathrm{~K}_{\mathrm{t}} \phi \rightarrow \mathrm{K}_{\mathrm{t}+1} \phi \quad$ (1, rule $\mathrm{RK}$ for $\mathrm{K}_{\mathrm{t}+1}$ : see Chellas, 1984, p. 19)

3. $\mathrm{K}_{\mathrm{t}} \phi \rightarrow \mathrm{K}_{\mathrm{t}+1} \mathrm{~K}_{\mathrm{t}} \phi \quad$ (axiom (A7))

4. $\mathrm{K}_{\mathrm{t}} \phi \rightarrow \mathrm{K}_{\mathrm{t}+1} \phi \quad$ (3, 2, Propositional Logic ).

Proof of Proposition 7. By Theorem 4.3 in van der Hoek (1993, p. 183) $\mathrm{K}_{\mathrm{KB45}}^{\mathrm{time}}+(\mathrm{A} 3)+(\mathrm{A} 4)$ is sound and complete with respect to the class of frames in $\mathcal{F}_{\text {KD45 }}$ that satisfy (R3) and (R4) (see the proof of Proposition 1). By Proposition 1, (R3) implies (P1) and (R4) implies (P2) (the proof does not rely on any properties of $\mathcal{K}_{\mathrm{t}}$ in particular the lack of reflexivity of $\mathcal{K}_{\mathrm{t}}$ is irrelevant). Thus the conjunction of (R3) and (R4) implies the conjunction of (P1) and (P2), which in turn is equivalent to (C) (again, the proof that the conjunction of (P1) and (P2) is equivalent to (C) does not rely on reflexivity of $\mathcal{K}_{\mathrm{t}}$ ). To see that, without reflexivity of $\mathcal{K}_{\mathrm{t}},(\mathrm{C})$ does not imply (R3) and (R4), consider the following KD45-frame: $\Omega=\{\alpha, \beta\}, \mathcal{K}_{0}(\alpha)=$ $\mathcal{K}_{0}(\beta)=\mathcal{B}_{0}(\alpha)=\mathcal{B}_{0}(\beta)=\{\beta\}$ and for all $t \geq 1, \mathcal{K}_{t}(\alpha)=\mathcal{K}_{t}(\beta)=\mathcal{B}_{t}(\alpha)=$ $\mathcal{B}_{\mathrm{t}}(\beta)=\{\alpha\}$. It is easily verified that, for every $t \in \mathbb{N}, \mathcal{K}_{\mathrm{t}}$ and $\mathcal{B}_{\mathrm{t}}$ are serial, transitive and euclidean and satisfy properties (R1) and (R2). Furthermore, $(C)$ is also (vacuously) satisfied. However, both (R3) and (R4) are violated. (R4) is violated because $\beta \in \mathcal{B}_{0}(\alpha)$ and $\alpha \in \mathcal{B}_{1}(\beta)$ and yet 
$\alpha \notin \mathcal{B}_{0}(\alpha)$. (R3) is violated because $\beta \in \mathcal{B}_{0}(\alpha)$ and yet there does not exist an $\omega \in \mathcal{B}_{0}(\alpha)$ such that $\beta \in \mathcal{B}_{1}(\omega)$.

\section{REFERENCES}

Alchourrón, Carlos, Peter Gärdenfors and David Makinson. 1985. 'On the logic of theory change: partial meet functions for contraction and revision'. Journal of Symbolic Logic, 50:510-30

Brown, P. M. 1976. 'Conditionalization and expected utility'. Philosophy of Science, 43:415-19

Chellas, Brian. 1984. Modal Logic: An Introduction. Cambridge University Press

Dekel, Eddie and Faruk Gul. 1996. 'Rationality and knowledge in game theory', mimeo, Northwestern University. (Forthcoming in Advances in Economic Theory, Seventh World Congress. Kreps D. M. and K. F. Wallis (eds.). Cambridge University Press.)

Friedman, Neil and Joseph Halpern. 1995. 'Modeling belief in dynamic systems. Part I: foundations', RJ 9965 (87924), IBM Research Division

Gärdenfors, Peter. 1988. Knowledge In Flux. MIT Press

Gärdenfors, Peter and David Makinson. 1988. 'Revisions of knowledge systems using epistemic entrenchment'. In Proceedings of the Second TARK Conference, pp. 83-95, M. Vardi (ed.). Morgan Kaufmann

Geanakoplos, John. 1994. 'Common knowledge'. In Handbook Of Game Theory, Vol. 2, pp. 1437-96. Robert Aumann and Sergiu Hart (eds). Elsevier

Halpem, Joseph. 1991. 'The relationship between knowledge, belief and certainty'. Annals of Mathematics and Artificial Intelligence, 4:301-22

Harman, Gilbert. 1986. Change In View: Principles Of Reasoning. MIT Press

Hintikka, Jaakko. 1962. Knowledge And Belief. Cornell University Press

van der Hoek, Wiebe. 1993. 'Systems for knowledge and belief'. Journal of Logic and Computation, 3:173-95

van der Hoek, Wiebe and J.-J. Ch. Meyer. 1995. Epistemic Logic For Artificial Intelligence And Computer Science. Cambridge University Press

Howson, Colin and Peter Urbach. 1989. Scientific Reasoning. Open Court

Jeffrey, Richard. 1983. The Logic Of Decision, 2nd edn. University of Chicago Press

Kraus, Sarit and Danile Lehmann. 1988. 'Knowledge, belief and time'. Theoretical Computer Science, 58:155-74

Lenzen, Wolfgang. 1978. 'Recent work in epistemic logic'. Acta Philosophica Fennica, 30:1-220

Maher, Patrick. 1993. Betting On Theories. Cambridge University Press

Mongin, Philippe. 1994. 'Some connections between epistemic logic and the theory of nonadditive probability'. In Patrick Suppes: Scientific Philosopher, Vol. 1, pp. 135-71. P. Humphreys (ed.). Kluwer

Osborne, Martin and Ariel Rubinstein. 1994. A Course In Game Theory. MIT Press

Piccione Michele and Ariel Rubinstein. 1995. 'On the interpretation of decision problems with imperfect recall'. Games and Economic Behavior, forthcoming

Stalnaker, Robert. 1984. Inquiry. MIT Press

Teller, P. 1973. 'Conditionalization and observation'. Synthese, 26:218-58 